\section{What is a Patient Navigator, and Should We Have Them in Major Cancer Centers?}

An October op-ed piece in The New York Times (http://www.nytimes.com/2009/10/10/ opinion/10DeVries.html?_r=1\&scp=1\&sq=mammologist $\&$ st=cse) introduced a new term into the medical lexicon: Mammologist. This title refers to a multidisciplinary breast cancer specialist who would serve as a facilitator specifically for patients with newly diagnosed breast cancer. The concept proposed was timely, with October marking Breast Cancer Awareness Month, although the goal of a mammologist - to ease patients through the steps of cancer care by being involved in every step of their treatment - is very similar to that of patient navigators.

The idea of a patient navigator is gaining currency at cancer centers around the country. Its rationale is based on recognition of the complicated layers cancer patients must negotiate from diagnosis through treatment and follow-up. Consider a "typical" woman with newly diagnosed breast cancer. She may already be under the care of a primary care physician and a gynecologist. She is sent for routine screening mammography and is told by the radiologist that she needs a biopsy based on the findings. Based on the biopsy results, she is referred to a breast surgeon and may also need to see a plastic surgeon, radiation oncologist, and medical oncologist. That's 7 doctors before even she gets to a second opinion!

Anyone who has tried to negotiate the current health care system knows how vexing it can be. Care at a comprehensive center-where radiology, surgery, and oncology specialists all reside under one roof and may even work through one scheduling officecan minimize some of the hurdles. But many patients are treated across multiple hospitals or have primary care medical teams that are distant from the cancer clinic. By distant, I mean either geographically or distant in terms of medical records, which could be as close as across the street but might as well be in a different time zone for the ease of transmission.

The availability of such complicated, sophisticated care is both a blessing and a curse. Although it affords patients access to true specialists, trained and ready to tackle their precise problem, it also creates logistical challenges that even the best health care consumers may stumble through. Data show that complex, multidisciplinary care can impair clinical outcomes because of the logistical difficulties. Quality initiatives have shown that physical distance to a cancer clinic can delay timely care. NCCN projects suggest that failure to meet quality benchmarks often arises because of the difficulties in transitioning patients to the next step in the care process. Even if the medical team and treatment algorithm state that the next step is obvious, getting the patient to that point can be a challenge.

Here is where a navigator could be invaluable. Through following institutional plans, the navigator could set up appointments, create individualized pathways for the patient, and serve as a concierge to steer the patient to the right desk, the next stop, and the planned destination. Nascent efforts are arising along these lines to help those known to face greater barriers to accessing health care, such as those without adequate health care coverage or financial resources, those with comorbid or mental illness, and those whose cultural or social circumstances might create alienation or separation from the established health care system.

But the same principles apply to more routine patients, too. It would be exciting to see major cancer centers pilot the notion of patient navigators, reaching out to specific groups of patients who have particularly daunting cancer diagnoses or where true, integrated multimodality care is essential. Creating institutional pathways and having navigators guide patients through these well-trafficked lanes would be an immense service, and would undoubtedly increase patient satisfaction, clarify many misunderstandings, and likely encourage better care. Historical data from NCCN institutions can benchmark the outcomes for quality of timely care. If modest pilot programs proved successful, others might move quickly to adopt broader navigator access. It certainly is an idea worth exploring.

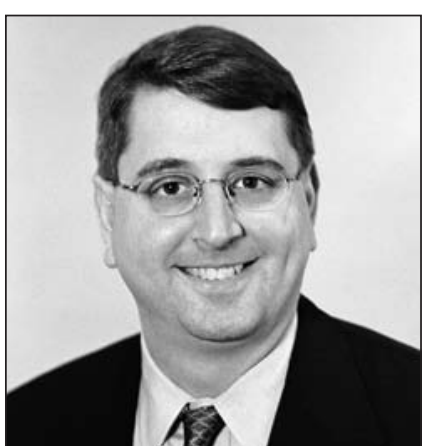

Harold J. Burstein, MD, PhD

Harold J. Burstein, MD, PhD, editor-in-chief of JNCCN, is an Associate Professor of Medicine at Harvard Medical School and a medical oncologist at Dana-Farber Cancer Institute and Brigham \& Women's Hospital. He is a clinician and clinical investigator specializing in breast cancer.

Dr. Burstein attended Harvard College and earned his MD at Harvard Medical School, where he also earned a PhD in immunology. He trained in internal medicine at Massachusetts General Hospital and was a fellow in medical oncology at Dana-Farber before joining the staff.

Dr. Burstein's clinical research interests include novel treatments for early- and advanced-stage breast cancer and studies of quality of life and health behavior among women with breast cancer. He has written widely on breast cancer in both traditional medical journals and on the web, including New England Journal of Medicine and Journal of Clinical Oncology. International committees focusing on cancer treatments that he has or continues to participate in include the NCCN Clinical Practice Guidelines Breast Cancer Panel, St. Gallen Breast Cancer Panel, CALGB Breast Cancer Committee, ASCO Health Services Research and Clinical Research Committees, the National Quality Forum Breast Cancer Technical Panel, and other ASCO expert panels.

The ideas and viewpoints expressed in this editorial are those of the author and do not necessarily represent any policy, position, or program of the NCCN. 